\title{
Patients with infective endocarditis and history of injection drug use in a Swedish referral hospital during 10 years
}

\author{
Anna Damlin ${ }^{1,2^{*}}$ and Katarina Westling ${ }^{3,4^{*}}$
}

\begin{abstract}
Background: Patients with injection drug use (IDU) have increased risk of developing infective endocarditis (IE). Previous studies have reported recurrent IE, increased duration of hospital stay, poor adherence and compliance as well as higher mortality and worse outcomes after surgery in the IDU-IE patient group. Further studies are needed to provide a basis for optimized care and prevention of readmissions in this population. This study aims to describe the clinical characteristics and outcomes among patients with IDU-IE.

Methods: Data of adults with IDU-IE and non-IDU-IE, treated between 2008 and 2017 at the Karolinska University Hospital in Stockholm were obtained from the Swedish National Registry of Infective Endocarditis. Clinical characteristics, microbiological results, treatment durations, results from echocardiography and in-hospital mortality were compared between the groups.

Results: Of the total 522 patients, 165 (32\%) had IDU-IE. Patients with IDU-IE were younger than the patients with non-IDU-IE (mean age IDU-IE: 41.6 years, SD 11.9 years; non-IDU-IE: 64.3 years, SD 16.4 years; $P<0.01$ ). No difference in distribution of gender was observed, $33 \%$ were females in both the IDU-IE and the non-IDU-IE group. History of previous IE (IDU-IE: $n=49$, 30\%; non-IDU-IE: $n=34,10 \% ; P<0.01$ ) and vascular phenomena (IDU-IE: $n=101,61 \%$; non-IDU-IE: $n=120,34 \%$; $P<0.01)$ were more common among patients with IDU-IE while prosthetic heart valves (IDU-IE: $n=12,7 \%$; non-IDU-IE: $n=83,23 \% ; P<0.01$ ) and known valvular disease (IDU-IE: $n=3,2 \%$; non-IDU-IE: $n=$ 78, 22\%; $P<0.01$ ) were more common among patients with non-IDU-IE. Aetiology of Staphylococcus aureus (IDU-IE: $n=123,75 \%$; non-IDU-IE: $n=118,33 \% ; P<0.01$ ) as well as tricuspid (IDU-IE: $n=91,55 \%$; non-IDU-IE: $n=23,6 \%$; $P<0.01$ ) or pulmonary valve vegetations (IDU-IE: $n=7,4 \%$; non-IDU-IE: $\mathrm{n}=2,1 \% ; P<0.01$ ) were more common in the IDU-IE group. The overall incidence of IDU-IE decreased during the study period, while the incidence of definite IE increased $(P<0.01)$.
\end{abstract}

Conclusions: This study presents that patients with IDU-IE were younger, less frequently treated with surgery and had higher prevalence of vascular phenomena and history of previous IE, aspects that are important for improved management of this population.

Keywords: Infective endocarditis, Injection drug use, Echocardiography

\footnotetext{
* Correspondence: anna.damlin@ki.se; katarina.westling@ki.se

'Department of Molecular Medicine and Surgery, Division of Clinical Physiology, Karolinska Institutet, SE-171 76 Stockholm, Sweden

${ }^{3}$ Department of Medicine Huddinge, Division of Infectious Diseases and

Dermatology, Karolinska Institutet, SE-141 86 Stockholm, Sweden

Full list of author information is available at the end of the article
} 


\section{Background}

Infective endocarditis (IE) is one of the infectious diseases with the highest morbidity and mortality [1-4]. In several countries, such as the United States, the incidences of IE have been reported as increasing [1-4]. One factor contributing to the increase of IE is the increasing incidence of injection drug use (IDU) [1-3]. People with IDU are at 100-fold increased risk of developing IE compared with the general population [1].

There are several mechanisms that can explain the increased risk of IE among patients with IDU: direct injury from injected substances, poor hygiene during injection, contaminated injection equipment, and physiological factors associated with IDU such as vasospasm and cardiac damage have also been described [2, 3, 5, 6]. IDUrelated IE (IDU-IE) more often present with right-sided vegetations, rather than left-sided. The exposure from injected substances causing endothelial damage has been reported as the mechanism behind the right-sided manifestations, which most often present as tricuspid valve vegetations $[3,6,7]$.

Previous studies have reported re-infections and relapses of IE, increased duration of hospital stay and poor adherence and compliance in the IDU-IE patient group [8-11]. Compared with patients with IE not related to IDU, cardiac surgery has been associated with higher mortality, worse risk-adjusted outcomes after surgery, and higher reoperation during the first 6 months after surgery in patients with IDU-IE $[9,12]$. The risks of continuing addiction and IDU in this patient group adds further complexity that needs to be taken into account in the management of patients with IDU-IE, however often inadequately addressed [13]. Further studies are needed to better understand the characteristics and outcomes among the patients with IDU-IE.

This study aimed to present the clinical characteristics and outcomes among the patients with IDU and definite IE admitted to a university hospital in Stockholm between 2008 and 2017. The hospital has a special ward for drug addicts with infectious diseases, which contributes to a high prevalence of IDU among the patients with IE, which was presented in a previous study [14]. This enables good comparability between patients with IDU-IE and patients with IE without IDU, referred to as non-IDU-IE.

\section{Methods}

\section{Study setting}

This study is based on data from patients with IDU and IE, admitted to the Karolinska University Hospital in Stockholm, Sweden. The hospital has a special ward for drug addicts with infectious diseases, with an uptake area covering Stockholm County Council with approximately 1.9 million adult (18 years and above) inhabitants.
The ward is operated by infectious diseases consultants and addiction psychiatrists. According to guidelines, most of the patients with IE at the hospital are discussed within an endocarditis team, comprising specialists in infectious diseases, cardiology, clinical physiology and thoracic surgery [15]. The majority of the echocardiographic examinations were conducted at the Department of Clinical Physiology, a unit specialized in cardiovascular imaging.

\section{Study design}

This study is registry-based on data from the Swedish National Registry of Infective Endocarditis (SRIE). The SRIE is a national registry established in 1995 , covering data from patients treated for possible or definite IE (ICD codes I33.0, I33.9, I38.9, and I39.8) in all Swedish departments for infectious diseases, with an estimated coverage of $70-80 \%$ [16]. In this study, data of patients with definite IE (patients with possible IE were excluded), treated at the Karolinska University Hospital in Stockholm, between 1 January 2008 and 31 December 2017 were obtained from the SRIE. Patients aged 18 years and above with registered history of IDU were selected for further analysis and compared with patients with non-IDU-IE. Age, gender, date of admission, clinical characteristics, congenital heart disease, comorbidities, results from blood cultures and cultures or polymerase chain reaction (PCR) amplification from material sampled during cardiac surgery, results from echocardiographic examinations (transthoracic echocardiography: TTE and transesophageal echocardiography: TEE), antibiotic treatment durations, and inhospital mortality were obtained from the SRIE. Coagulase-negative staphylococci (CoNS) were grouped including Staphylococcus lugdunensis and Staphylococcus epidermidis. The review of data from the registry was performed by one person (AD) and discussed with a specialist in infectious diseases (KW). All methods were performed in accordance with the relevant guidelines and regulations [15].

\section{Statistical methods}

Continuous variables were described as means and standard deviations (SDs) or median and 25th and 75th percentiles and categorical variables as proportions (percentages). Mann-Whitney's test was used for comparisons of means. For comparisons of medians, quantile regression was used. For comparisons of categorical variables, chi-squared test was conducted for values $\geq 5$ and the two-sided Fisher's exact test for values $<5$. Logistic regression models were used to analyse in-hospital mortality in different patient groups. Variations over time were analysed using linear regression. In all analyses, $P$ values $<0.05$ (two-tailed) were considered statistically 
significant. Analyses were performed using STATA software (version 15.1 Stata Corp., College Station, Texas, USA).

\section{Results}

In total, 522 patients were registered with definite IE at the Karolinska University Hospital between 2008 and 2017, of these 165 (32\%) had history of IDU. The incidence of definite IE registered at the Karolinska University Hospital increased during the study period from 2.52 (2008) to 4.21 cases (2017) per 100,000 adult (18 years and older) inhabitants in Stockholm county $(P<0.01)$. Contrasting, there was an overall decrease in incidence of IDU-IE registered at the hospital between 2008 and $2017(P<0.01)$, however, an initial increase between 2008 and 2011 was seen from 0.58 to 1.34 cases per 100,000 adult inhabitants $(P<0.01$, Fig. 1$)$, but from 2012 to 2017, there was a decrease in incidence from 1.14 to 0.89 cases per 100,000 adult inhabitants in Stockholm county $(\mathrm{P}<0.01$, Fig. 1$)$. Further, the rate of patients with IDU-IE were lower during the winter compared to the other seasons $(P=0.01)$.

The clinical characteristics of the patients with IDU-IE and non-IDU-IE are presented in Table 1. About two thirds of the included patients with IDU-IE were male (110 patients, 67\%), which was the same percentage as among non-IDU-IE patients. Mean age among the patients with IDU-IE was 41.6 years (SD 11.9 years), which was mean 22.7 years younger than patients with non-IDUIE (mean age 64.3 years, SD 16.4 years; $P<0.01$ ). A majority of the patients with IDU-IE (101 patients, 61\%) presented with vascular phenomena, which was more common than among patients with non-IDU-IE (Table 1). More specifically, spondylitis and pulmonary septic emboli (both presented as symptomatic complications) were more common among IDU-IE compared with patients with non-IDU-IE (spondylitis $n=25,15 \%$ vs $n=30,9 \%$; $P=0.02$, pulmonary septic emboli $n=55,33 \%$ vs $n=11$, $3 \% ; P<0.01$ ). Further, history of previous IE was more common among the patients with IDU-IE, while prosthetic heart valves, known valvular disease, cardiovascular implantable electronic device (CIED)-associated IE were more common among patients with non-IDU-IE (Table 1). During the 6-month follow up, 3 (2\%) of the patients with IDU-IE had reported recurrence of IE (i.e. relapse or re-infection), not significantly different from the non-IDU-IE group $(n=5,1 \%$; $P=0.72$ ). Of the patients with recurrent IE in the IDU-IE group, one had relapse (i.e. subsequent episode caused by the same microorganism) and the other two were re-infected (i.e. subsequent episode caused by different microorganisms). Of the patients with recurrent IE in the non-IDU-IE group, four had relapse and one was re-infected.

Of the included patients with IDU-IE, 155 (94\%) had positive blood cultures. One of the patients that had negative blood culture had positive polymerase chain reaction (PCR) from heart valve surgery (methicillin susceptible Staphylococcus aureus - MSSA) (Table 2 and Table 3). Aetiology of $S$. aureus was significantly more common among patients with IDU-IE compared with patients with non-IDU-IE (IDU-IE: $n=123,75 \%$; nonIDU-IE: $n=118,33 \% ; P<0.01$ ). Of the 522 patients, 274 (52\%) underwent both TTE and TEE (IDU-IE: $n=79$,

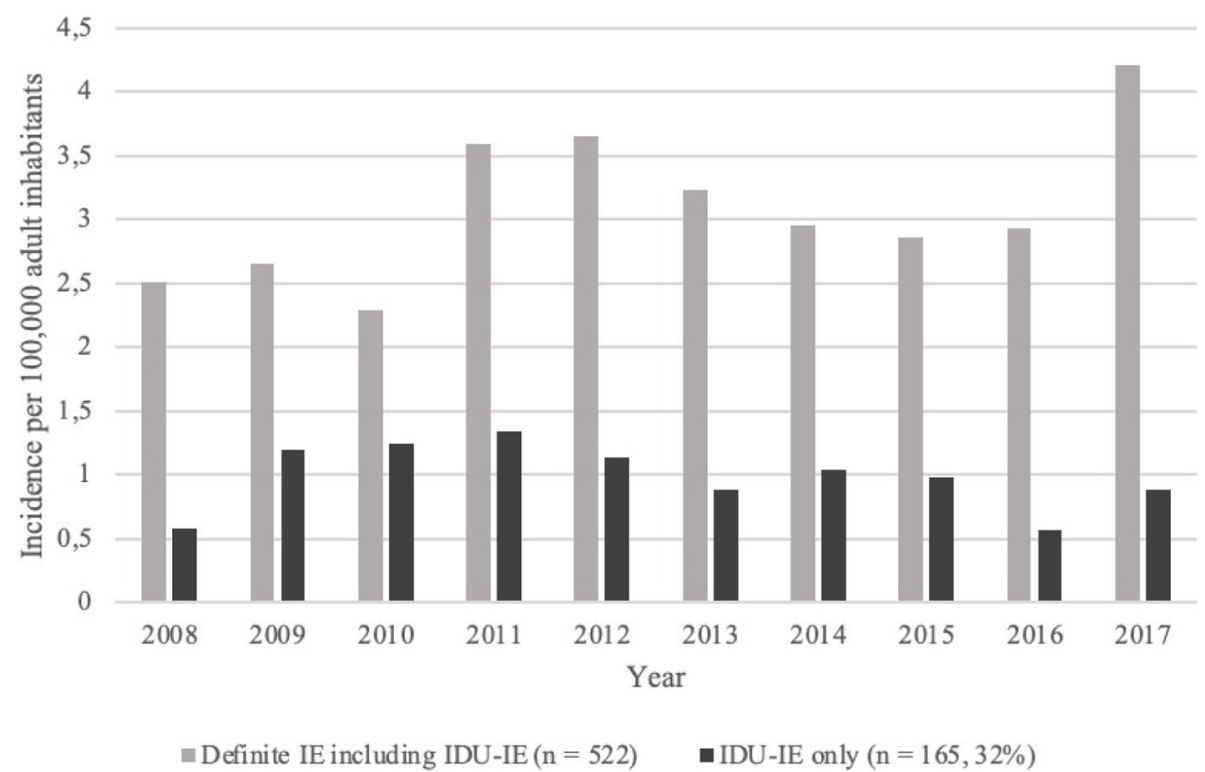

Fig. 1 Incidences of definite IE and IDU-IE admitted between 2008 and 2017. Incidences are presented in number of cases per 100,000 adult inhabitants in Stockholm county each year. Abbreviations: IDU, injection drug use; IE, infective endocarditis 
Table 1 Characteristics of the patients with IDU-IE and non-IDU-IE admitted between 2008 and 2017

\begin{tabular}{|c|c|c|c|}
\hline Clinical characteristics & IDU-IE $(n=165(\%))$ & Non-IDU IE $(n=357(\%))$ & $P$ value \\
\hline Women & $55(33)$ & $119(33)$ & 1.00 \\
\hline Men & $110(67)$ & $238(67)$ & 1.00 \\
\hline Age, mean ( \pm SD); median ( 25 th and 75 th percentiles) & $41.6(11.9) ; 44(31,50)$ & $64.3(16.4) ; 67(56,76)$ & $<0.01$ \\
\hline \multicolumn{4}{|l|}{ Predisposing factors } \\
\hline Bicuspid aortic valve & $0(0)$ & $20(6)$ & $<0.01$ \\
\hline Prosthetic valve & $12(7)$ & $83(23)$ & $<0.01$ \\
\hline CIED & $2(1)$ & $38(11)$ & $<0.01$ \\
\hline Rheumatic heart disease & $0(0)$ & $2(1)$ & 1.00 \\
\hline Congenital heart disease & $0(0)$ & $8(2)$ & 0.06 \\
\hline History of IE & $49(30)$ & $34(10)$ & $<0.01$ \\
\hline Known valvular disease & $3(2)$ & $78(22)$ & $<0.01$ \\
\hline Heart failure before or under IE treatment & $19(12)$ & $45(13)$ & 0.72 \\
\hline \multicolumn{4}{|l|}{ Patients fulfilling Duke's major criteria } \\
\hline Blood culture positive for IE & $144(87)$ & $304(85)$ & 0.78 \\
\hline Echocardiography positive for $\mathrm{IE}^{\mathrm{a}}$ & $165(100)$ & $287(80)$ & $<0.01$ \\
\hline \multicolumn{4}{|l|}{ Patients fulfilling Duke's minor criteria } \\
\hline Fever & $150(91)$ & $304(85)$ & 0.07 \\
\hline Vascular phenomena & $101(61)$ & $120(34)$ & $<0.01$ \\
\hline Spondylitis & $25(15)$ & $30(9)$ & 0.02 \\
\hline Pulmonary septic emboli & $55(33)$ & $11(3)$ & $<0.01$ \\
\hline New heart murmur & $20(12)$ & $63(18)$ & 0.11 \\
\hline Immunological phenomenon & $2(1)$ & $19(5)$ & 0.03 \\
\hline \multicolumn{4}{|l|}{ Outcomes } \\
\hline Surgery & $27(16)$ & $121(34)$ & $<0.01$ \\
\hline In-hospital mortality & $7(4)$ & $27(8)$ & 0.15 \\
\hline Relapse during follow up ${ }^{b}$ & $1(0)$ & $4(1)$ & 1.00 \\
\hline Re-infected during follow up ${ }^{b}$ & $2(1)$ & $1(0)$ & 0.24 \\
\hline
\end{tabular}

an total, 101 (61\%) of the IDU-IE patients underwent transthoracic echocardiography and 137 (83\%) patients underwent transoesophageal echocardiography during hospital stay. ${ }^{b}$ Follow up within 6 months from discharge. Significant $P$ values are marked in bold font. Abbreviations: CIED cardiovascular implantable electronic device; IDU injection drug use; IE infective endocarditis; $n$ number of patients; $S D$ standard deviation

48\%; non-IDU-IE: $n=195,55 \%), 168$ (32\%) underwent TEE only (IDU-IE: $n=58,35 \%$; non-IDU-IE: $n=110$, $31 \%)$ and 58 (11\%) underwent TTE only (IDU-IE: $n=22$, 13\%; non-IDU-IE: $n=36,10 \%)$. Of the patients with IDU-IE, 94\%, compared with $80 \%$ of the patients with non-IDU-IE $(\mathrm{P}<0.01)$, had findings consistent with IE during examination with echocardiography, the most common manifestation was tricuspid valve vegetation (91 patients, 55\%), followed by mitral valve vegetation (47 patients, 28\%) and aortic valve vegetation (33 patients, 20\%) (Table 4). A significantly higher prevalence of tricuspid and pulmonary valve vegetations was seen among the patients with IDU-IE compared with the non-IDU-IE patients who had higher prevalence of aortic- and mitral valve vegetations as well as abscess and CIED-associated IE (Table 4). Multiple valve vegetations were similar among patients with IDU-IE $(n=27(16 \%))$ and non-IDU-IE ( $n=46(13 \%), P=0.29)$.

Significantly less of the IDU-IE patients was treated with surgery; 27 (16\%), compared with 121 (34\%) among the non-IDU-IE patients $(P<0.01)$. The rate of patients with IDU-IE treated with surgery did not change significantly during the study period $(P=0.06)$. The indications for surgery were assessed from the European guidelines for the management of IE [15]. The most common indications for surgery in the IDU-IE-group were heart failure $(n=19,12 \%)$, followed by vegetation $(n=14,8 \%)$ while vegetation $(n=56,16 \%)$ followed by heart failure ( $n=42,12 \%)$ were the most common indications for surgery in the non-IDU-IE-group.

The most common single valve surgery performed in the IDU-IE group were biological aortic valve replacement 
Table 2 Aetiology obtained from blood- and valve culture among patients with IDU-IE and non-IDU-IE

\begin{tabular}{|c|c|c|c|}
\hline & IDU-IE & Non-IDU-IE & $P$ value \\
\hline Blood culture results ${ }^{\mathrm{a}}(\mathrm{n}(\%))$ & $144(87)$ & $336(94)$ & $<0.01$ \\
\hline Staphylococcus aureus (MSSA) & $110(67)$ & $116(32)$ & $<0.01$ \\
\hline Enterococcus faecalis & $14(8)$ & $34(10)$ & 0.70 \\
\hline Viridans group streptococci & $9(5)$ & $94(26)$ & $<0.01$ \\
\hline S. aureus (MRSA) & $3(2)$ & $3(1)$ & 0.39 \\
\hline Bacillus cereus & $3(2)$ & $0(0)$ & - \\
\hline CoNS & $2(1)$ & $22(6)$ & 0.01 \\
\hline Group A streptococci & $1(1)$ & $1(0)$ & 0.53 \\
\hline Group B streptococci & $0(0$ & $11(3)$ & - \\
\hline Group G streptococci & $1(1)$ & $6(2)$ & 0.44 \\
\hline Klebsiella spp & $1(1)$ & $0(0)$ & - \\
\hline Candida spp. & $0(0)$ & $1(0)$ & - \\
\hline Unspecified funghi & $1(1)$ & $1(0)$ & 0.53 \\
\hline Polymicrobial blood cultures & $11(7)$ & $7(2)$ & $<0.01$ \\
\hline MSSA and E. faecalis & $4(2)$ & $0(0)$ & - \\
\hline E. faecalis and unspecified funghi & $1(1)$ & $0(0)$ & - \\
\hline MSSA and aerobic gramnegative stave & $1(1)$ & $0(0)$ & - \\
\hline MSSA and candida & $1(1)$ & $0(0)$ & - \\
\hline MSSA and group G streptococci & $1(1)$ & $0(0)$ & - \\
\hline MSSA and unspecified funghi & $1(1)$ & $0(0)$ & - \\
\hline MSSA and viridans group streptococci & $1(1)$ & $1(0)$ & 0.53 \\
\hline Viridans group streptococci and CoNS & $1(1)$ & $0(0)$ & - \\
\hline Blood culture negative & $8(5)$ & $19(5)$ & 0.82 \\
\hline Bacteria in valve culture from cardiac surgery & $9(5)$ & $17(5)$ & 0.73 \\
\hline Staphylococcus aureus (MSSA) & $5(3)$ & $7(2)$ & 0.53 \\
\hline Unspecified funghi & $2(1)$ & $1(0)$ & - \\
\hline CoNS & $1(1)$ & $2(1)$ & 1.00 \\
\hline Enterococcus faecalis & $1(1)$ & $2(1)$ & 1.00 \\
\hline
\end{tabular}

${ }^{a}$ Single bacteria in blood culture. All patients in the IDU-IE group with positive valve culture had positive blood culture. Two of the patients in the non-IDU-IE group with positive valve culture had negative blood cultures. Of the patients with positive valve culture in the IDU-IE group, one had bacteria that differed between blood culture (MSSA) and valve culture (unspecified funghi). Of the patients with positive valve culture in the non-IDU-IE group, one had bacteria that differed between blood culture (MSSA) and valve culture (viridans group streptococci). Abbreviations: CoNS coagulase negative staphylococci; $E$ enterococcus; IDU injection drug use; IE infective endocarditis; MRSA methicillin resistant Staphylococcus aureus; MSSA methicillin susceptible Staphylococcus aureus; $n$ number of patients; PCR polymerase chain reaction; $S$ staphylococcus; spp. species

Table 3 Aetiology obtained from valve PCR among patients with IDU-IE and non-IDU-IE

\begin{tabular}{llll}
\hline & IDU-IE & Non-IDU-IE & P value \\
\hline Bacteria in valve PCR from cardiac surgery (n (\%)) & $\mathbf{1 9 ( 1 2 )}$ & $\mathbf{1 4}(\mathbf{4})$ & $2(1)$ \\
Staphylococcus aureus (MSSA) & $15(9)$ & $1(0)$ & $<.01$ \\
CoNS & $2(1)$ & 0.01 \\
Enterococcus faecalis & $2(1)$ & 0.24 \\
Viridans group streptococci & $0(0)$ & $6(2)$ & - \\
\hline
\end{tabular}

One patient in the IDU-IE group and three patients in the non-IDU-IE group had positive valve PCR (IDU-IE: MSSA; non-IDU-IE: viridans group streptococci, unspecified gram positive bacteria and Streptococcus bovis) and negative blood culture, all other patients with positive valve PCR had positive blood cultures. Among the patients with positive valve PCR had positive blood cultures, the bacteria found in blood culture were the same as those found in valve PCR. Abbreviations: CoNS coagulase negative staphylococci; IDU injection drug use; IE infective endocarditis; MSSA methicillin susceptible Staphylococcus aureus; $n$ number of patients; $P C R$ polymerase chain reaction 
Table 4 Manifestations detected by echocardiography among patients with IDU-IE and non-IDU-IE

\begin{tabular}{|c|c|c|c|}
\hline All manifestations & $\begin{array}{l}\text { IDU-IE } \\
(n=165(100 \%))\end{array}$ & $\begin{array}{l}\text { Non-IDU-IE } \\
(n=287(80 \%))\end{array}$ & $P$ value \\
\hline Aortic valve vegetation & $33(20)$ & $171(48)$ & $<0.01$ \\
\hline Mitral valve vegetation & $47(28)$ & $162(45)$ & $<0.01$ \\
\hline Tricuspid valve vegetation & $91(55)$ & $23(6)$ & $<0.01$ \\
\hline Pulmonary valve vegetation & $7(4)$ & $2(1)$ & $<0.01$ \\
\hline CIED-associated IE & $1(1)$ & $25(7)$ & $<0.01$ \\
\hline Abscess & $4(2)$ & $27(8)$ & 0.03 \\
\hline \multicolumn{4}{|l|}{ Multiple valve vegetations } \\
\hline Aortic and mitral valve vegetation & $11(7)$ & $31(9)$ & \multirow[t]{7}{*}{0.43} \\
\hline Aortic and tricuspid valve vegetation & $5(3)$ & $3(1)$ & \\
\hline Aortic and pulmonary valve vegetation & $1(1)$ & $0(0)$ & \\
\hline Mitral and tricuspid valve vegetation & $5(3)$ & $5(1)$ & \\
\hline Mitral and pulmonary valve vegetation & $1(1)$ & $0(0)$ & \\
\hline Tricuspid and pulmonary valve vegetation & $4(2)$ & $1(0)$ & \\
\hline CIED and tricuspid valve vegetation & $0(0)$ & $6(2)$ & \\
\hline
\end{tabular}

Among the patients listed with, for instance, aortic valve vegetation, some patients had vegetations also on other valves and thus were listed also under the topic "multiple valve vegetations". Significant $P$ values are marked in bold font. Abbreviations: CIED cardiovascular implantable electronic device; IDU injection drug use, IE infective endocarditis

$(n=6)$ followed by biological tricuspid valve replacement $(n=5)$, and one removal of tricuspid valve vegetation. Eight surgical procedures included multiple valve replacement of which 5 cases included tricuspid valve replacement. Among the patients with IDU-IE treated with surgery, none were re-operated during hospital stay nor during follow-up (up to 6 months). One patient with IDUIE caused by $E$ faecalis, that was not treated with surgery during hospital stay was operated during follow-up due to prosthetic (mechanical) mitral valve dehiscence, that was inserted 14 years prior to the IE diagnosis. Among the patients with IDU-IE treated with surgery, one died during hospital stay but none died during follow-up after discharge from hospital. In-hospital mortality was similar among the patients treated with surgery and the patients not treated with surgery, both in the IDU-IE group (1 $(4 \%)$ of the 27 patients treated with surgery died during hospital stay and $6(5 \%)$ of the 132 patients not treated with surgery died during hospital stay, $P=1.00$ ) and in the non-IDU-IE group (12 (10\%) of the 121 patients treated with surgery died during hospital stay and $15(6 \%)$ of the 236 patients not treated with surgery died during hospital stay, $P=0.23$ ).

In-hospital mortality was similar among patients with IDU-IE $(n=7(4 \%))$, and those with non-IDU-IE (nonIDU-IE $n=27(8 \%), P=0.15)$. Among the patients with IDU-IE, the most common cause of death was heart failure $(n=4)$ followed by cardiac arrest $(n=1)$, intracerebral haemorrhage $(n=1)$ and liver failure $(n=1)$. Also among patients with non-IDU-IE, the most common cause of death was heart failure $(n=15)$. Among patients with S. aureus aetiology, in-hospital mortality was higher among patients with non-IDU-IE compared with the patients with IDU-IE $(n=18(15 \%)$ and $n=5(4 \%)$, respectively, $P=0.01)$. In the IDU-IE group, $80(70 \%)$ of the patients with $S$. aureus aetiology had right-sided IE, and 35 (30\%) had left sided IE. In the non-IDU-IE group, 20 $(18 \%)$ of the patients with $S$. aureus aetiology had rightsided IE, and 95 (83\%) had left sided IE. Hence, of the patients with $S$. aureus aetiology, right sided IE was more common in the IDU-IE group (OR 10.74; $P<0.01)$. In the IDU-IE group, $3(4 \%)$ of the patients with $S$. aureus aetiology and right-sided IE died during hospital stay, which was similar to the in-hospital mortality among the left-sided IE $(n=2(6 \%)$; OR $0.64 ; P=$ $0.64)$. In the non-IDU-IE group, $2(10 \%)$ of the patients with $S$. aureus aetiology and right-sided IE died during hospital stay, which was similar to the in-hospital mortality among the left-sided IE $(n=14 \quad(15 \%)$; OR 0.63 ; $P=0.73)$.

The duration of hospital stay was similar among patients with non-IDU-IE (median 32 days, interquartile range (IQR) 20 days) compared with patients with IDUIE (median 32 days, IQR 14 days; $P=1.00$ ). The duration of antibiotic treatment was longer in the non-IDU-IE group (median 30 days, IQR 10 days) compared with the IDU-IE group (median 28 days, IQR 12 days; $P=0.02$ ). Patients with left-sided IDU-IE caused by $S$. aureus had similar antibiotic treatment durations (median 28 days, IQR 12 days) as patients with $S$. aureus and right-sided IDU-IE (median 28 days, IQR 9 days; P 1.00). The patients that died during hospital stay had shorter duration 
of antibiotic treatment (median 23 days, IQR 13 days) compared with the patients that survived (median 30 days, IQR 11 days; $P<0.01$ ).

\section{Discussion}

This study presents history of previous IE was more common in the IDU-IE group, however a lower extent of the patients with IDU-IE had prosthetic heart valves and known valvular disease compared with those with non-IDU-IE. The rate of patients with positive echocardiography was higher among patients with IDU-IE compared with non-IDU-IE, which could possibly be explained by the higher rate of right-sided valve vegetations in the IDU-IE group, which can be easier to identify also with TTE. Another reason could be the younger age and less comorbidities in the IDU-IE group compared with the non-IDU-IE group, which could have contributed to more frequent use of TEE in this group, which has higher sensitivity and specificity for IE, compared with TTE.

The patients with IDU-IE were younger than the patients with non-IDU-IE. Supportive to our results, previous studies of IDU-IE present younger populations compared to patients with non-IDU-IE [2, 3, 8, 17]. The population of patients with IE is known to have a domination of male gender, the ratio of male to female patients are commonly presented as $3: 1$ or $2: 1$ [17-19]. However, Wurcel et al., presented a prevalence of female gender of $40.9 \%$ among IDU-IE and even higher in the age group of 15-34-year-old patients with IDU-IE (3). The population of IDU-IE patients in our study had a similar rate of female patients in the age group 15-34 years as in that study. This finding suggest that the demographics of patients with IDU-IE are shifting towards a population of younger, female patients compared with previously reported demographics of the IDU-IE population [3, 17].

Previous studies have described an increasing incidence of IE in general, and of IDU-IE specifically $[1,8]$. In our study, the incidence of definite IE registered at the Karolinska University Hospital increased during the study period, but the incidence varied among the patients with IDU-IE. There was an increase in incidence of IDU-IE registered at the hospital between 2008 and 2011 but from 2011 to 2017, there was an overall decrease. Further, the rate of patients with IDU-IE were lower during the winter compared to the other seasons, which according to our knowledge, has not been presented before. Seasonal changes in infectious diseases have been described before [20]. Most bacteria tend to increase with rising temperature [20], which could possibly explain the lower rate of IE during the winter season. The decrease in incidence of IDU-IE after 2011 might be explained by a syringe exchange programme that was introduced in Stockholm at that time. Such programs have shown to reduce HIV and hepatitis infections, but also have been suggested to decrease bacterial infections among IDU, such as IE [2].

$S$. aureus was the most common aetiology among the patients with IDU-IE, and significantly more common compared with patients with non-IDU-IE. This finding is supported by previous studies that have shown $S$. aureus was the most common aetiology among IDU-IE [1, 10, 21]. The high prevalence of $S$. aureus among the IDU-IE may explain the higher prevalence of vascular phenomena such as spondylitis and pulmonary septic emboli among the IDUIE compared with the non-IDU-IE, findings that are supported by Lassen et al. [21]. In our study, patients with $S$. aureus aetiology and non-IDU-IE had higher in-hospital mortality compared with the patients with $S$. aureus aetiology and IDU-IE. Further, left-sided IE was more common among the patients with S. aureus aetiology and non-IDUIE. Previous studies of patients with IE have presented higher in-hospital mortality among patients with $S$. aureus aetiology and left-sided IE, compared with right-sided IE $[22,23]$. Further, polymicrobial infection was more common among the patients with IDU-IE compared with the patients with non-IDU-IE. This finding is supported by a study by Sousa et al. [24]. Polymicrobial aetiology together with prior IE, left-sided IE, intracardiac complications, and stroke were presented as risk factors for 6-month mortality among patients with IDU-IE, according to a multinational study of 7616 patients with IE [25].

In this study, patients with IDU-IE were less treated with surgery compared to patients with non-IDU-IE. This is supported by the multinational study that presented that surgery was less frequently performed in patients with IDU-IE [25]. The rate of patients with IDUIE treated with surgery did not change significantly during the study period. The in-hospital mortality was similar among patients treated with surgery and those not treated with surgery, both in the IDU-IE and the nonIDU-IE group. However, a previous study presented that IDU-IE was associated with a higher hazard of death or reoperation between 90 and 180 days after first surgery, and that reoperation were less common among nonIDU-IE [9]. Such increased risk in the IDU-IE population should be taken into account for the management of these patients.

In our study, the in-hospital mortality was similar among patients with IDU-IE and non-IDU-IE, although the relatively low absolute numbers of patients could explain the absence of a significant difference. However, supportive to our results are two studies from the U.S., one that showed hospital mortality did not differ between IDU-IE and non-IDU-IE patients and one that presented less hospital mortality among IDU-IE $[1,8]$. On the contrary, both studies presented a longer 
duration of hospital stay among IDU-IE which was not the case in this study $[1,8]$.

History of previous IE among the admitted patients, were more common in the IDU-IE group compared with the patients with non-IDU-IE. This has been described before $[1,8,10]$. Lassen et al., described $29 \%$ of the patients with IDU-IE had recurrence during follow-up [21]. Relapse of IDU has been described as a major risk factor of reinfection in patients with IDU-IE. This increases the risks of potential need for surgical treatment, and death [13]. Hence, treatment of addiction is crucial in patients with IDU-IE [13].

\section{Limitations}

This study was based on data from the Swedish National Registry of Infective Endocarditis. Information about some parameters such as surgical criteria were limited. Surgical criteria were only registered for patients that underwent surgery. Hence, it was not possible to make comparisons of mortality, or other outcomes between patients that had indication for surgery but did not undergo surgery and those with indication that underwent surgery. The registry did include information about some comorbidities, although not all comorbidities needed for a quantification of overall comorbidity using the Charlson comorbidity index. As data obtained from the SRIE were anonymized and did not include any personal data, some patients might have been admitted more than once, and thus included in the registry as a new patient. This could possibly have contributed to competitive risks.

\section{Conclusions}

History of previous IE was more common in the IDU-IE group, in which the patients were younger and had less comorbidities compared with the non-IDU-IE group. However, spondylitis and pulmonary septic emboli were more common in the IDU-IE group. S. aureus was the most common aetiology among the patients with IDUIE, and significantly more common compared with patients with non-IDU-IE. Further, polymicrobial infection was more common among the patients with IDU-IE compared with the patients with non-IDU-IE. Patients with IDU-IE were less treated with surgery compared to patients with non-IDU-IE. Conclusively, as patients with IDU-IE tend to have higher recurrence of IE compared with patients with non-IDU-IE and tend to present with severe complications and polymicrobial infections, these factors should be taken into account for the management of these patients.

\section{Authors' contributions}

A.D. and K.W. designed the study. A.D. analyzed the data and A.D. and K.W. interpreted it. A.D. was a major contributor in writing the manuscript, both authors revised it. Both authors read and approved the final manuscript.

\section{Funding}

A.D.: No funding. K.W.: No funding. Open Access funding provided by Karolinska Institute.

\section{Availability of data and materials}

The data that support the findings of this study were taken from the SRIE, but restrictions apply to the availability of these data, which were used under license for the current study, and so are not available publicly. However, data are available from the first author upon reasonable request and with permission of the SRIE.

\section{Ethics approval and consent to participate}

The study was approved by the Regional Ethics Review Board, Stockholm, Sweden (diary number K 2018-6018). Informed consents were given from the patients. None of the patients were dead when included for registration in the SRIE, hence no written informed consent from the legally authorized representatives/next of kin of patients were obtained. Data obtained from the SRIE were anonymized and did not include any personal data.

\section{Consent for publication}

Not applicable.

\section{Competing interests}

The authors declare that they have no competing interests.

\section{Author details}

${ }^{1}$ Department of Molecular Medicine and Surgery, Division of Clinical Physiology, Karolinska Institutet, SE-171 76 Stockholm, Sweden. ${ }^{2}$ Department of Clinical Physiology, Karolinska University Hospital, A8:01, Eugeniavägen 3, SE-171 76 Stockholm, Sweden. ${ }^{3}$ Department of Medicine Huddinge, Division of Infectious Diseases and Dermatology, Karolinska Institutet, SE-141 86 Stockholm, Sweden. ${ }^{4}$ Department of Infectious Diseases, Karolinska University Hospital Huddinge, SE-141 86 Stockholm, Sweden.

Received: 17 November 2020 Accepted: 16 February 2021

Published online: 02 March 2021

\section{References}

1. Rudasill SE, Sanaiha Y, Mardock AL, Khoury H, Xing H, Antonios JW, et al. Clinical outcomes of infective endocarditis in injection drug users. J Am Coll Cardiol. 2019;73(5):559-70.

2. Keeshin SW, Feinberg J. Endocarditis as a marker for new epidemics of injection drug use. Am J Med Sci. 2016;352(6):609-14.

3. Wurcel AG, Anderson JE, Chui KK, Skinner S, Knox TA, Snydman DR, et al. Increasing Infectious Endocarditis Admissions Among Young People Who Inject Drugs. Open Forum Infect Dis. 2016;3(3):ofw157.

4. Pant S, Patel NJ, Deshmukh A, Golwala H, Patel N, Badheka A, et al. Trends in infective endocarditis incidence, microbiology, and valve replacement in the United States from 2000 to 2011. J Am Coll Cardiol. 2015;65(19):2070-6.

5. Frontera JA, Gradon JD. Right-side endocarditis in injection drug users: review of proposed mechanisms of pathogenesis. Clin Infect Dis. 2000;30(2): 374-9.

6. Shih C-J, Chu H, Chao P-W, Lee Y-J, Kuo S-C, Li S-Y, et al. Long-term clinical outcome of major adverse cardiac events in survivors of infective endocarditis. Circulation. 2014;130(19):1684-91.

7. Akinosoglou K, Apostolakis E, Koutsogiannis N, Leivaditis V, Gogos CA. Rightsided infective endocarditis: surgical management. Eur J Cardiothorac Surg. 2012:42(3):470-9.

8. Hartman L, Barnes E, Bachmann L, Schafer K, Lovato J, Files DC. Opiate injection-associated infective endocarditis in the southeastern United States. Am J Med Sci. 2016:352(6):603-8.

9. Shrestha NK, Jue J, Hussain ST, Jerry JM, Pettersson GB, Menon V, et al. Injection drug use and outcomes after surgical intervention for infective endocarditis. Ann Thorac Surg. 2015;100(3):875-82.

10. Alagna L, Park LP, Nicholson BP, Keiger AJ, Strahilevitz J, Morris A, et al. Repeat endocarditis: analysis of risk factors based on the international 
collaboration on endocarditis - prospective cohort study. Clin Microbiol Infect. 2014;20(6):566-75.

11. Rabkin DG, Mokadam NA, Miller DW, Goetz RR, Verrier ED, Aldea GS. Longterm outcome for the surgical treatment of infective endocarditis with a focus on intravenous drug users. Ann Thorac Surg. 2012;93(1):51-7.

12. Geirsson A, Schranz A, Jawitz O, Mori M, Feng L, Zwischenberger BA, et al. The evolving burden of drug use associated infective endocarditis in the United States. Ann Thorac Surg. 2020;110(4):1185-92.

13. Rosenthal ES, Karchmer AW, Theisen-Toupal J, Castillo RA, Rowley CF. Suboptimal addiction interventions for patients hospitalized with injection drug use-associated infective endocarditis. Am J Med. 2016;129(5):481-5.

14. Damlin A, Westling K, Maret E, Stålsby Lundborg C, Caidahl K, Eriksson MJ. Associations between echocardiographic manifestations and bacterial species in patients with infective endocarditis: a cohort study. BMC Infect Dis. 2019;19(1):1052

15. Habib G, Lancellotti P, Antunes MJ, Bongiorni MG, Casalta JP, Del Zotti F, et al. 2015 ESC guidelines for the management of infective endocarditis: the task force for the Management of Infective Endocarditis of the European Society of Cardiology (ESC). Endorsed by: European Association for CardioThoracic Surgery (EACTS), the European Association of Nuclear Medicine (EANM). Eur Heart J. 2015;36(44):3075-128.

16. Olaison L. Årsrapport endokarditregistret 2018: Svenska Endokarditregistret; 2017 (SWEDISH). https://infektion.net/wp-content/uploads/2019/09/endoka rditregistret_18_web.pdf. Accessed 2 Oct 2020.

17. Miró JM. del Río a, Mestres CA. Infective endocarditis in intravenous drug abusers and HIV-1 infected patients. Infect Dis Clin N Am. 2002;16(2):273-95.

18. Sambola A, Fernandez-Hidalgo N, Almirante B, Roca I, Gonzalez-Alujas T, Serra $B$, et al. Sex differences in native-valve infective endocarditis in a single tertiary-care hospital. Am J Cardiol. 2010;106(1):92-8.

19. Aksoy O, Meyer LT, Cabell CH, Kourany WM, Pappas PA, Sexton DJ. Gender differences in infective endocarditis: pre- and co-morbid conditions lead to different management and outcomes in female patients. Scand J Infect Dis, 2007;39(2):101-7.

20. Schwab F, Gastmeier P, Hoffmann P, Meyer E. Summer, sun and sepsis-the influence of outside temperature on nosocomial bloodstream infections: a cohort study and review of the literature. PLoS One. 2020;15(6):e0234656.

21. Lassen H, Nielsen SL, Gill SUA, Johansen IS. The epidemiology of infective endocarditis with focus on non-device related right-sided infective endocarditis: a retrospective register-based study in the region of southern Denmark. Int J Infect Dis. 2020;95:224-30.

22. López J, Fernández-Hidalgo N, Revilla A, Vilacosta I, Tornos P, Almirante B, et al. Internal and external validation of a model to predict adverse outcomes in patients with left-sided infective endocarditis. Heart. 2011; 97(14):1138-42.

23. Murray RJ. Staphylococcus aureus infective endocarditis: diagnosis and management guidelines. Intern Med J. 2005;35(Suppl 2):S25-44.

24. Sousa C, Botelho C, Rodrigues D, Azeredo J, Oliveira R. Infective endocarditis in intravenous drug abusers: an update. Eur J Clin Microbiol Infect Dis. 2012; 31(11):2905-10.

25. Pericàs JM, Llopis J, Athan E, Hernández-Meneses M, Hannan MM, Murdoch DR, et al. Prospective cohort study of infective endocarditis in people who inject drugs. J Am Coll Cardiol. 2021;77(5):544-55.

\section{Publisher's Note}

Springer Nature remains neutral with regard to jurisdictional claims in published maps and institutional affiliations.

Ready to submit your research? Choose BMC and benefit from:
- fast, convenient online submission
- thorough peer review by experienced researchers in your field
- rapid publication on acceptance
- support for research data, including large and complex data types
- gold Open Access which fosters wider collaboration and increased citations
- maximum visibility for your research: over 100M website views per year
At BMC, research is always in progress.
Learn more biomedcentral.com/submissions

